\title{
Tumor immunohistochemistry and preoperative magnetic resonance imaging features predict local recurrence of giant cell tumor of bone following intralesional curettage
}

\author{
YIFENG HE $^{1 *}$, YU ZHOU $^{1 *}$, JI ZHANG $^{1}$, FEI YUAN ${ }^{2}$, JUN WANG $^{3}$, \\ LIANJUN DU ${ }^{1}$, QI ZHOU ${ }^{3}$, JING LIANG ${ }^{3}$ and XIAOYI DING ${ }^{1}$
}

\begin{abstract}
Departments of ${ }^{1}$ Radiology and ${ }^{2}$ Pathology, Ruijin Hospital, Shanghai Jiao Tong University School of Medicine;
${ }^{3}$ Shanghai Institute of Traumatology and Orthopedics, Shanghai Key Laboratory for The Prevention and Treatment of Bone and Joint Diseases, Ruijin Hospital, Shanghai Jiao Tong University School of Medicine, Shanghai 200025, P.R. China
\end{abstract}

Received March 16, 2017; Accepted February 6, 2018

DOI: $10.3892 / \mathrm{ol} .2018 .9778$

\begin{abstract}
Giant cell tumor of bone (GCTB) is among the most prevalent types of tumor of the bone in East and Southeast Asia. The high rate of local recurrence following intralesional curettage poses a challenge for the clinical treatment of GCTB. In the present study, the expression of matrix metalloproteinase-9 (MMP-9), vascular endothelial growth factor (VEGF), receptor activator of nuclear factor- $\kappa \mathrm{B}$ (RANK) and RANK ligand (RANKL) in GCTB was investigated by immunohistochemical staining. A correlation between expression and preoperative magnetic resonance imaging (MRI) features was identified. The patients were followed up for $\geq 2$ years after intralesional curettage. The rates of local recurrence were compared among different groups. A total of 74 cases of GCTB in the proximal tibia or distal femur were investigated. MRI features were retrospectively examined by correlation analysis. The expression of MMP-9 was demonstrated to be associated with cystic changes and the 'paint brush borders' sign $(\mathrm{P}<0.05)$, and positively associated with the expression of RANKL $(\mathrm{P}<0.05)$ and VEGF $(\mathrm{P}<0.05)$. Cystic changes, the 'paint brush borders' sign and adjacent soft tissue invasion were associated with high rates of local recurrence $(\mathrm{P}<0.05)$ and were therefore identified as significant risk factors. Pathologically, the 'paint brush borders' sign was demonstrated to be indicative of local invasion of the bone. These findings may be useful for predicting local recurrence of GCTB, and may provide important insight into the preoperative MRI features and molecular expression patterns of GCTB tumors.
\end{abstract}

Correspondence to: Dr Xiaoyi Ding, Department of Radiology, Ruijin Hospital, Shanghai Jiao Tong University School of Medicine, 197 Rui Jin Er Road, Shanghai 200025, P.R. China

E-mail: dingxiaoyi@hotmail.com

*Contributed equally

Key words: preoperative magnetic resonance imaging, giant cell tumor of bone, local recurrence, immunohistochemistry, prognostic factor

\section{Introduction}

Giant cell tumor of bone (GCTB) is characterized by a large number of osteoclastic giant cells uniformly distributed amongst mononuclear spindle-like stromal cells and other rounded monocytes $(1,2)$. It is among the most prevalent tumors of the bone in East and Southeast Asia, accounting for $\sim 20 \%$ of all cases (3-5). It is classified as an intermediate tumor (6) with characteristic locally invasive behavior leading to osteolysis $(1,7,8)$. GCTB is mainly treated by curettage; however, clinically, the high rate of local recurrence following curettage (13-65\%) poses a major challenge for the treatment of GCTB $(9,10)$.

Factors contributing to the prognosis of local recurrence of GCTB require investigation in order to achieve local control and guidance for surgical approaches. In the present study, literature concerning molecular prognostic factors for local recurrence of GCTB was reviewed to identify various potential prognostic factors. High activities of matrix metalloproteinase (MMP) and vascular endothelial growth factor (VEGF) have been linked to the biological aggressiveness of GCTB $(11,12)$. Kumta et al (13) demonstrated that elevated levels of VEGF and MMP-9 in GCTB were positively correlated with local recurrence. In bone tumors, co-overexpression of receptor activator of nuclear factor- $\mathrm{KB}$ (RANK) and RANK ligand (RANKL) was identified as a potential discriminating factor for a poor prognosis (14). The expression of RANKL affected the proliferation of neoplastic GCTB cells in another study (15). Based on these studies, it has been hypothesized that GCTB expressing elevated levels of these proteins may be more prone to recurrence.

As a non-invasive method, magnetic resonance imaging (MRI) is valuable for the diagnosis and evaluation of bone tumors, owing to its heightened sensitivity to soft tissue disease and multiplanar image acquisition (16). MRI may provide information regarding tumor margins and soft tissue extension, as well as cystic changes (17-19). Biological aggressiveness and poor prognoses are associated with the aforementioned molecular expression patterns of GCTB (11-13), and may also be evaluated through preoperative MRI. However, to the best of our knowledge, no previous studies have analyzed 
the association between molecular expression patterns, MRI features and the prognosis of GCTB.

The present study aimed to investigate the association between prognostic protein expression and preoperative MRI features, and its value in predicting the local recurrence of GCTB. The present study was inspired by appeals for the establishment of a novel multiple-perspective evaluation system (20). Furthermore, elucidation of the role of the expression of the aforementioned proteins in the local recurrence of GCTB will provide insight into the clinical value of investigating these molecular mechanisms.

\section{Patients and methods}

Patients. The present study was approved by the Institutional Review Board of Ruijin Hospital, Shanghai Jiao Tong University School of Medicine (Shanghai, China). Written informed consent was obtained from all patients prior to enrolment in the study. All enrolled patients had been diagnosed with histopathologically-confirmed GCTB of the proximal tibia or distal femur. MRI and computerized tomography scans of all patients were obtained and analyzed prior to surgery.

Currently, intralesional excision is the preferred treatment of GCTB, rather than en bloc resection, although the latter is associated with reduced recurrence with the compromise of limb function. Both treatments are selective for patients at Ruijin Hospital.

To examine the factors associated with local recurrence, patients with $\geq 2$ years of follow-up information available subsequent to intralesional curettage treatment, which was performed consistently by orthopedic specialists in Ruijin Hospital, were selected for retrospective study. A total of 69 patients were prospectively enrolled in the present study between January 2005 and October 2015. A total of 35 male and 39 female patients were eligible for inclusion in the present study. The median age was 29 years (range, 17-64 years). To exclude the impact of increased complexity of surgical treatment, 5 patients with pathological fractures and 4 patients with soft tissue masses were excluded from the present study.

Between March 2013 and July 2016, 36 patients (18 male and 18 female; mean age, 32.56 years; median age, 28.5 years; age range, 21-61 years) with GCTB around the knee were enrolled for investigation. Immunohistochemistry (IHC) features, including the protein expression of VEGF, MMP-9, RANKL and RANK, were studied. A total of 22 of these patients overlapped with the former group; en bloc resection was performed in the remaining 14 patients, 2 of whom were enrolled for investigation on the pathological basis of specific preoperative MRI features. Adjacent tissues obtained in seven cases as a negative control.

Immunohistochemistry (IHC). IHC was performed on formalin-fixed $\left(23-26^{\circ} \mathrm{C}\right.$ for $\left.24 \mathrm{~h}\right)$, paraffin-embedded tumor tissue samples, cut into $3-\mu \mathrm{m}$ thick sections. The sections were deparaffinized with xylene, rehydrated in a descending ethanol series (100\% for $5 \mathrm{~min}$; $100 \%$ for $5 \mathrm{~min}$; $95 \%$ for $5 \mathrm{~min}$; $95 \%$ for $5 \mathrm{~min}$; $85 \%$ for $3 \mathrm{~min}$ and $75 \%$ for $2 \mathrm{~min}$ ), and treated using an automated immunostainer Bench Mark XT (Ventana Medical Systems, Inc., Tucson, AZ, USA) following antigen retrieval with citrate buffer (cat. no. ab64214; Abcam, Cambridge, UK) of $\mathrm{pH} 6.0$, microwaved on high power until boiling $\left(93^{\circ} \mathrm{C}\right)$ for
$5 \mathrm{~min}$. The slides were kept warm by heating for $10 \mathrm{~min}$ at low power $\left(4^{\circ} \mathrm{C}\right)$. The coplin jar was left to sit in the microwave for at least $20 \mathrm{~min}$. The sections were incubated with the following primary antibodies for $1 \mathrm{~h}$ at $37^{\circ} \mathrm{C}$ : MMP-9 (dilution, 1:1,000; cat. no. ab38898), VEGF receptor 1 (dilution, 1:250; cat. no. Y103), RANK (dilution, 1:50; cat. no. 64C1385) and RANKL (dilution, 1:100; cat. no. 12A668; all Abcam). The polymeric detection system, ultra View Universal DAB Detection kit(VentanaMedical Systems, Inc.), was then used according to the manufacturer's protocol. Finally, the sections were counter-stained with Gill's modified hematoxylin (6 g/l; cat. no. GH316; Sigma-Aldrich; Merck KGaA, Darmstadt, Germany) at room temperature for $17 \mathrm{~min}$, and then cover-slipped with $\mathrm{EUKITT}^{\circledR}$ (ORSAtec $\mathrm{GmbH}$, Bobingen, Germany) mounting media. Mouse brain tissue (15 days old wild-type mouse embryonic brain; Department of Laboratory Animal Sciences, Shanghai Jiao tong University School of Medicine, Shanghai, China) was used as a positive tissue control for the anti-VEGF antibody, and RAW 264.7 cells [Type Culture Collection of the Chinese Academy of Sciences; cells were cultured in Dulbecco's modified Eagle's medium (cat. no. 12800017) and $10 \%$ fetal bovine serum (cat. no. 10100147) (both from Gibco; Thermo Fisher Scientific, Inc., Waltham, MA, USA) in an atmosphere containing $5 \% \mathrm{CO}_{2}$ at $37^{\circ} \mathrm{C}$ ] were used as the positive control for the other antibodies. IHC on adjacent tissues in the absence of the primary antibody were used as a negative control. The sections were analyzed with an Olympus light microscope (Olympus Corporation, Tokyo, Japan) using x10 and $\mathrm{x} 40$ objectives. Independent experienced pathologists FY and QZ, blinded to the clinical details of the individual patients, evaluated the immunoreactivity of VEGF, RANKL, RANK and MMP-9, using a scoring system. The expression levels of VEGF, RANKL, RANK and MMP-9 were semi-quantified using a visual grading system based on the extent of staining: grade 0 , virtually no immunoreactivity; grade 1 , patchy to diffuse weak immunoreactivity; grade 2 , patchy to diffuse moderate immunoreactivity; and grade 3, patchy to diffuse strong immunoreactivity, as previously described $(21,22)$. However, according to the results of the IHC analysis, there were very few cases of grade 0 and grade 3 ; therefore, $0-1$ cases were classified into a low-grade group, and grade 2-3 cases into a high-grade group.

Imaging procedures. MRI was performed using a 1.5-T superconducting whole-body imager (SIGNA; GE Healthcare, Chicago, IL, USA) with dedicated extremity coils. A combination of axial, sagittal and coronal images was obtained using the following sequences: Spin-echo T1-weighted (TR range/TE range, 450-600 ms/15-20 ms), fast spin-echo T2-weighted (TR range/TE range, 3,500-4,000/80-120 ms) and fat-suppressed fast spin-echo T2-weighted (TR range/TE range, 3,500-4,000/80-120 ms). The field of view, slice thickness and inter slice gap varied depending on the region of interest and tumor size. The slice thickness was $<5 \mathrm{~mm}$ and inter slice gap was 0.5 to $2.0 \mathrm{~mm}$. The imaging matrix ranged from $192 \times 256$ to $256 \times 256$ pixels. For follow-up, the patients underwent routine anterior-posterior and lateral plain imaging.

Objective features and groups. A total of 4 imaging features, namely, cystic changes, the 'paint brush borders' sign, peritzmoral edema and adjacent soft tissue invasion, 
Table I. Patient characteristics.

\begin{tabular}{ll}
\hline Characteristic & Number $(\%)$ \\
\hline Age & \\
$\leq 28$ years & $37(50.00)$ \\
$>28$ years & $37(50.00)$ \\
Sex & \\
Male & $35(47.30)$ \\
Female & $39(52.70)$ \\
Location & \\
Proximal tibia & $34(45.96)$ \\
Distal femur & $40(54.04)$ \\
'Paint brush borders' sign & \\
Present & $31(41.89)$ \\
Absent & $43(58.11)$ \\
Peritumoral edema & \\
Grade A & $38(51.35)$ \\
Grade B & $36(48.65)$ \\
Adjacent soft tissue invasion & \\
Yes & $53(71.62)$ \\
No & $21(28.38)$ \\
Cystic changes & \\
Yes & $51(68.92)$ \\
No & $23(31.08)$ \\
\hline
\end{tabular}

were evaluated and interpreted by 3 senior musculoskeletal radiologists (LD, JZ and XD, with 17, 22 and 25 years of experience, respectively) until a diagnostic consensus was reached. However, if a mutual consensus could not be reached, the majority opinion was used. GCTB cases were classified into positive and negative groups according to the aforementioned preoperative imaging features. GCTB margins are observed as well-defined, irregularly penetrating margins. The "paint brush borders' sign is defined as penetrating irregular margins with a 'paint brush' appearance protruding towards the bone. This is visible on MRI scans as they offer a stark contrast between the high signal intensity of the bone marrow and the low signal intensity of tumor tissue. T1-weighted images provide a good demonstration of manifesting irregular protrusions compared with other sequences. Patients were classified as positive for the 'paint brush borders' if such margins were noted, irrespective of tumor number and shape. Cystic changes indicate liquefactive necrosis. This feature may be associated with a high cellular or protein content, and manifests as a homogeneous high signal intensity on T2-weighted images. Peritumoral edema is identifiable by a very high signal intensity on fat-suppressed MRI scans, which are used for the evaluation of joint effusion and edema. GCTBs were classified into 2 grades according to the presence of edema: Grade A, high signal intensity on fat-suppressed T2-weighted images and grade B, minimally increased signal intensity on fat-suppressed T2-weighted images. In GCTB, adjacent soft tissue invasion is indicative of tumor aggressiveness. Specifically, this feature is an indication of peripheral soft tissue involvement, coupled with loss of bone cortex, defined by low signal intensity on MRI scans (23).
Intralesional curettage procedure. All 60 patients were treated with intralesional curettage, which was performed by senior orthopedic surgeons. The tumor tissue was removed with a curette following creation of a wide cortical window. The remainder of the tumor cavity was eliminated with a high-speed burr. Phenol (5\%) was applied to the borders of the cavity with cotton-tipped applicators at room temperature for 6 min, and then neutralized with $75 \%$ ethanol twice in 33 cases at room temperature. The remaining 27 cases were treated without phenol. Finally, the tumorcavity was carefully packed with polymethyl methacrylate filling. Surgical specimens were obtained from 2 selected patients treated with en bloc resection.

Follow-up and recurrence. All patients were re-examined by radiography or MRI annually for $\geq 2$ years, regardless of their symptomatic status. For patients who were unable to undergo re-examination in Ruijin hospital, telephone, written or home visit follow-up was performed. Enlargement of the radiolucent zone was considered a reliable indicator of possible local recurrence. Recurrent tumor sex hibited high signal intensity around the polymethyl methacrylate filling on T2-weighted images. Patients were also re-examined immediately if any abnormal pain or swelling occurred following surgery.

Statistical analysis. Statistical analyses were performed using SPSS (version 22.0; IBM Corp., Armonk, NY, USA). Categorical variables are presented as the percentage and absolute number of patients. The associations between dichotomous attributes and local recurrence were analyzed using the $\chi^{2}$ test. Fisher's exact test was used if the number of cases was $<40$. Independent factors were determined by multivariate logistic regression analysis. $\mathrm{P}<0.05$ was considered to indicate a statistically significant difference.

\section{Results}

Patient characteristics. All patient groups were classified according to the preoperative MRI features. The positive rates of the 'paint brush borders' sign, peritumoral edema, cystic changes and adjacent soft tissue invasion were $41.89,51.35,68.92$ and $71.62 \%$, respectively. Tissue specimens from 36 patients were investigated by IHC. The clinical characteristics of all enrolled patients are detailed in Tables I and II.

Associations between preoperative MRI features and protein expression. High-grade MMP-9 expression was positively associated with cystic changes $(\mathrm{P}<0.05)$ and the 'paint brush borders' sign $(\mathrm{P}<0.05)$. MMP-9 expression also associated with the expression of RANKL $(\mathrm{P}<0.05)$ and VEGF $(\mathrm{P}<0.05$; Table III). However, VEGF, RANK and RANKL expression were not associated with any preoperative MRI features of GCTB (P>0.05; Figs. 1 and 2).

Follow-up and local recurrence. A total of 55 patients $(91.67 \%)$ treated within tralesional curettage were successfully followed up, while the remaining 5 patients were lost to follow-up. A total of 32 patients did not experience recurrence. A total of 23 patients (41.18\%) were diagnosed with recurrent GCTB; 20 of these patients $(86.96 \%)$ experienced recurrence 
Table II. Comparison of patients in terms of preoperative MRI characteristics and immunohistochemistry scores.

\begin{tabular}{|c|c|c|c|c|c|c|c|c|c|c|}
\hline \multirow[b]{2}{*}{ Patient number } & \multirow[b]{2}{*}{ Surgical approach } & \multirow[b]{2}{*}{ Local recurrence } & \multicolumn{4}{|c|}{$\begin{array}{l}\text { Preoperative MRI } \\
\text { feature }\end{array}$} & \multicolumn{4}{|c|}{ Immunohistochemistry staining score } \\
\hline & & & $\mathrm{PE}$ & ASTI & PS & $\mathrm{CC}$ & RANKL & MMP-9 & VEGF & RANK \\
\hline 1 & Curettage & No & B & No & No & Yes & 1 & 2 & 2 & 1 \\
\hline 2 & Curettage & No & A & Yes & No & No & 2 & 1 & 1 & 0 \\
\hline 3 & Curettage & No & A & Yes & No & Yes & 2 & 1 & 0 & 1 \\
\hline 4 & Curettage & No & B & Yes & No & Yes & 2 & 2 & 1 & 1 \\
\hline 5 & Curettage & No & B & Yes & No & No & 1 & 1 & 1 & 1 \\
\hline 6 & Curettage & No & B & No & No & No & 1 & 1 & 1 & 1 \\
\hline 7 & Curettage & No & A & Yes & No & No & 1 & 1 & 2 & 2 \\
\hline 8 & Curettage & No & B & No & No & No & 1 & 1 & 0 & 0 \\
\hline 9 & Curettage & No & A & No & No & No & 2 & 1 & 1 & 1 \\
\hline 10 & Curettage & No & B & Yes & No & No & 1 & 1 & 2 & 1 \\
\hline 11 & Curettage & No & B & Yes & Yes & No & 1 & 0 & 3 & 0 \\
\hline 12 & Curettage & Yes & A & No & Yes & Yes & 2 & 2 & 3 & 2 \\
\hline 13 & Curettage & Yes & A & Yes & No & Yes & 1 & 1 & 0 & 2 \\
\hline 14 & Curettage & Yes & A & Yes & No & Yes & 2 & 2 & 2 & 2 \\
\hline 15 & Curettage & Yes & B & Yes & No & Yes & 2 & 0 & 1 & 1 \\
\hline 16 & Curettage & Yes & A & Yes & No & Yes & 1 & 1 & 2 & 1 \\
\hline 17 & Curettage & Yes & B & Yes & Yes & Yes & 2 & 2 & 2 & 0 \\
\hline 18 & Curettage & Yes & A & Yes & Yes & Yes & 1 & 0 & 0 & 1 \\
\hline 19 & Curettage & Yes & A & Yes & Yes & Yes & 3 & 2 & 1 & 0 \\
\hline 20 & Curettage & Yes & B & Yes & Yes & Yes & 1 & 0 & 1 & 1 \\
\hline 21 & Curettage & Yes & B & Yes & Yes & No & 3 & 2 & 2 & 1 \\
\hline 22 & Curettage & Yes & A & Yes & Yes & Yes & 2 & 2 & 2 & 2 \\
\hline 23 & Resection & l & B & Yes & No & Yes & 1 & 0 & 1 & 0 \\
\hline 24 & Resection & I & B & Yes & No & Yes & 1 & 2 & 1 & 2 \\
\hline 25 & Resection & I & B & Yes & Yes & Yes & 3 & 2 & 2 & 1 \\
\hline 26 & Resection & I & B & No & No & No & 2 & 0 & 1 & 1 \\
\hline 27 & Resection & I & B & Yes & No & Yes & 2 & 1 & 1 & 2 \\
\hline 28 & Resection & I & B & No & No & Yes & 1 & 1 & 2 & 1 \\
\hline 29 & Resection & / & B & No & No & Yes & 2 & 1 & 2 & 1 \\
\hline 30 & Resection & I & A & Yes & Yes & No & 1 & 1 & 1 & 0 \\
\hline 31 & Resection & I & B & Yes & No & No & 2 & 1 & 1 & 2 \\
\hline 32 & Resection & I & A & Yes & No & No & 2 & 1 & 1 & 3 \\
\hline 33 & Resection & I & B & Yes & Yes & No & 2 & 2 & 2 & 1 \\
\hline 34 & Resection & / & A & Yes & Yes & Yes & 1 & 2 & 1 & 1 \\
\hline 35 & Resection & I & B & Yes & Yes & Yes & 1 & 0 & 1 & 2 \\
\hline 36 & Resection & / & B & Yes & No & Yes & 2 & 2 & 2 & 2 \\
\hline
\end{tabular}

MRI, magnetic resonance imaging; PE, peritumoral edema; ASTI, adjacent soft tissue invasion; PS, 'paint brush borders' sign; CC, cystic changes; A/B, grade A/B; RANKL, receptor activator of nuclear factor- $\kappa \mathrm{B}$ ligand; RANK, receptor activator of nuclear factor- $\kappa \mathrm{B}$; VEGF, vascular endothelial growth factor; MMP-9, matrix metalloproteinase-9.

within 2 years (mean, 14.7 months). Of the remaining 3 recurrent cases, 2 experienced recurrence after 5 years and one after 8 years. The follow-up period ranged between 2 and 10 years (mean, 5.61 years).

Preoperative MRI features and protein expression for predicting local recurrence. Among the 55 patients successfully followed up, 37 exhibited cystic changes, and of these,
22 patients $(59.46 \%)$ developed recurrence. Of the 18 patients who did not exhibit cystic changes, only 1 (5.56\%) experienced recurrence $\left(\chi^{2}=14.461 ; \mathrm{P}<0.05\right)$. Furthermore, the "paint brush borders' sign was observed in 21 patients (Figs. 3 and 4); $16(76.19 \%)$ of these patients experienced relapse during follow-up (Fig. 4). Of the 34 patients who did not exhibit the 'paint brush borders' sign, only 7 (20.59\%) developed local recurrence $\left(\chi^{2}=16.496 ; P<0.05\right)$. Furthermore, adjacent soft 
Table III. Association between MRI features and protein expression.

\begin{tabular}{|c|c|c|c|c|c|c|c|c|}
\hline \multirow[b]{3}{*}{ MRI feature } & \multicolumn{8}{|c|}{ High-grade immunohistochemistry } \\
\hline & \multicolumn{2}{|c|}{ VEGF } & \multicolumn{2}{|c|}{ MMP-9 } & \multicolumn{2}{|c|}{ RANK } & \multicolumn{2}{|c|}{ RANKL } \\
\hline & Number (\%) & P-value & Number $(\%)$ & P-value & Number $(\%)$ & P-value & Number (\%) & P-value \\
\hline $\begin{array}{l}\text { 'Paint brush borders' } \\
\text { positive group }\end{array}$ & $7(53.8)$ & 0.31 & $8(61.54)$ & $0.03^{\mathrm{a}}$ & $3(23.1)$ & 0.71 & $7(53.8)$ & 1.00 \\
\hline $\begin{array}{l}\text { 'Paint brush borders' } \\
\text { negative group }\end{array}$ & $8(34.8)$ & & $5(21.74)$ & & $8(34.8)$ & & $12(52.2)$ & \\
\hline $\begin{array}{l}\text { Cystic change } \\
\text { positive group }\end{array}$ & $10(45.5)$ & 0.73 & $11(50.0)$ & $0.40^{\mathrm{a}}$ & $8(36.4)$ & 0.47 & $12(54.5)$ & 1.00 \\
\hline $\begin{array}{l}\text { Cystic change } \\
\text { negative group }\end{array}$ & $5(35)$ & & $2(14.3)$ & & $3(21.4)$ & & $7(50)$ & \\
\hline $\begin{array}{l}\text { Peritumoral edema high } \\
\text { level group }\end{array}$ & $5(35.7)$ & 0.73 & $5(35.7)$ & 1.00 & $6(42.9)$ & 0.27 & $8(57.1)$ & 0.74 \\
\hline $\begin{array}{l}\text { Peritumoral edema low } \\
\text { level group }\end{array}$ & $10(45.5)$ & & $8(36.4)$ & & $5(22.7)$ & & $11(50.0)$ & \\
\hline $\begin{array}{l}\text { Soft tissue invasion } \\
\text { positive group }\end{array}$ & $11(39.3)$ & 0.69 & $11(39.3)$ & 0.68 & $10(35.7)$ & 0.39 & $15(53.6)$ & 1.00 \\
\hline $\begin{array}{l}\text { Soft tissue invasion } \\
\text { negative group }\end{array}$ & $4(50.0)$ & & $2(25.0)$ & & $1(12.5)$ & & $4(50)$ & \\
\hline High-grade MMP-9 & $9(69.2)$ & $0.02^{\mathrm{a}}$ & & & & & & \\
\hline Low-grade MMP-9 & $6(26.1)$ & & & & & & & \\
\hline High-grade RANK & $5(45.5)$ & 1.00 & $5(45.5)$ & 0.48 & & & & \\
\hline Low-grade RANK & $10(40.0)$ & & $8(32.0)$ & & & & & \\
\hline High-grade RANKL & $9(47.4)$ & 0.52 & $10(52.6)$ & $0.04^{\mathrm{a}}$ & $7(36.8)$ & 0.48 & & \\
\hline Low-grade RANKL & $6(35.3)$ & & $3(17.6)$ & & $4(23.5)$ & & & \\
\hline
\end{tabular}

${ }^{a} \mathrm{P}<0.05$. MRI, magnetic resonance imaging; VEGF, vascular endothelial growth factor; MMP-9, matrix metalloproteinase-9; RANK, receptor activator of nuclear factor- $\kappa \mathrm{B}$; RANKL, receptor activator of nuclear factor- $\mathrm{kB}$ ligand.
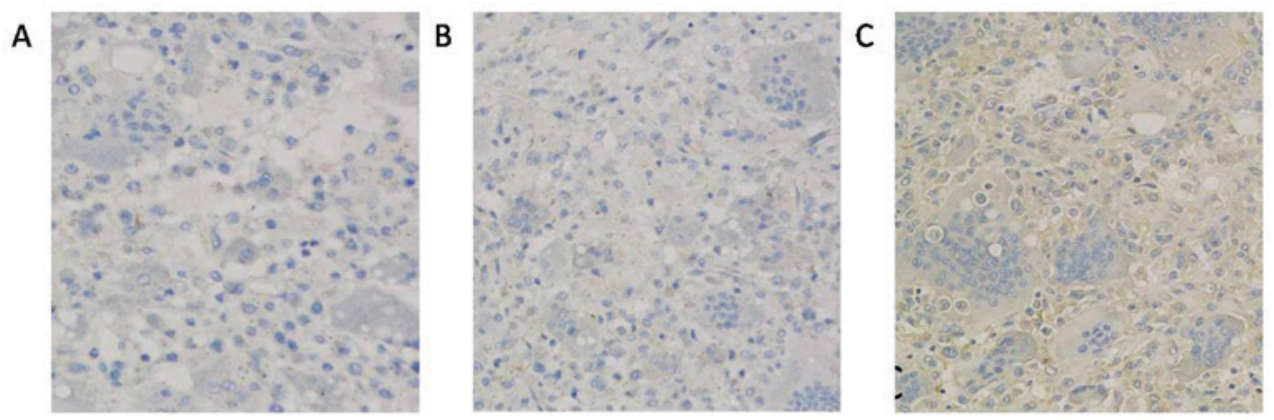

D

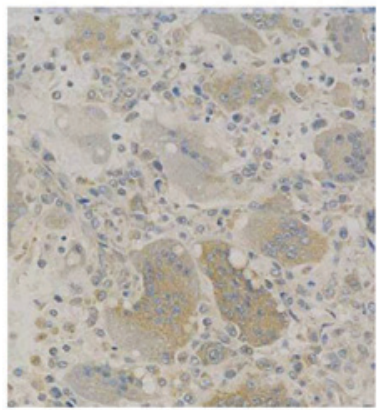

E

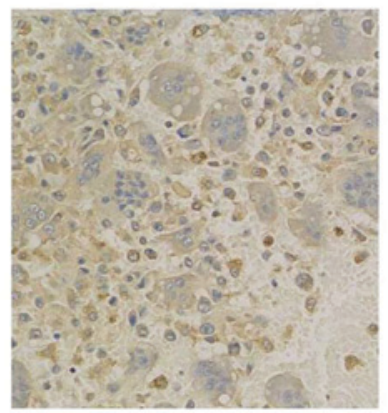

Figure 1. Immunohistochemical staining of receptor activator of nuclear factor- $\kappa$ B ligand. (A) Negative control test. (B) IHC grade 0 , virtually no immunoreactivity. (C) IHC grade 1, diffuse weak immunoreactivity. (D) IHC grade 2, diffuse moderate immunoreactivity. (E) IHC grade 3, diffuse strong immunoreactivity. Magnification, x200. IHC, immunohistochemistry. 

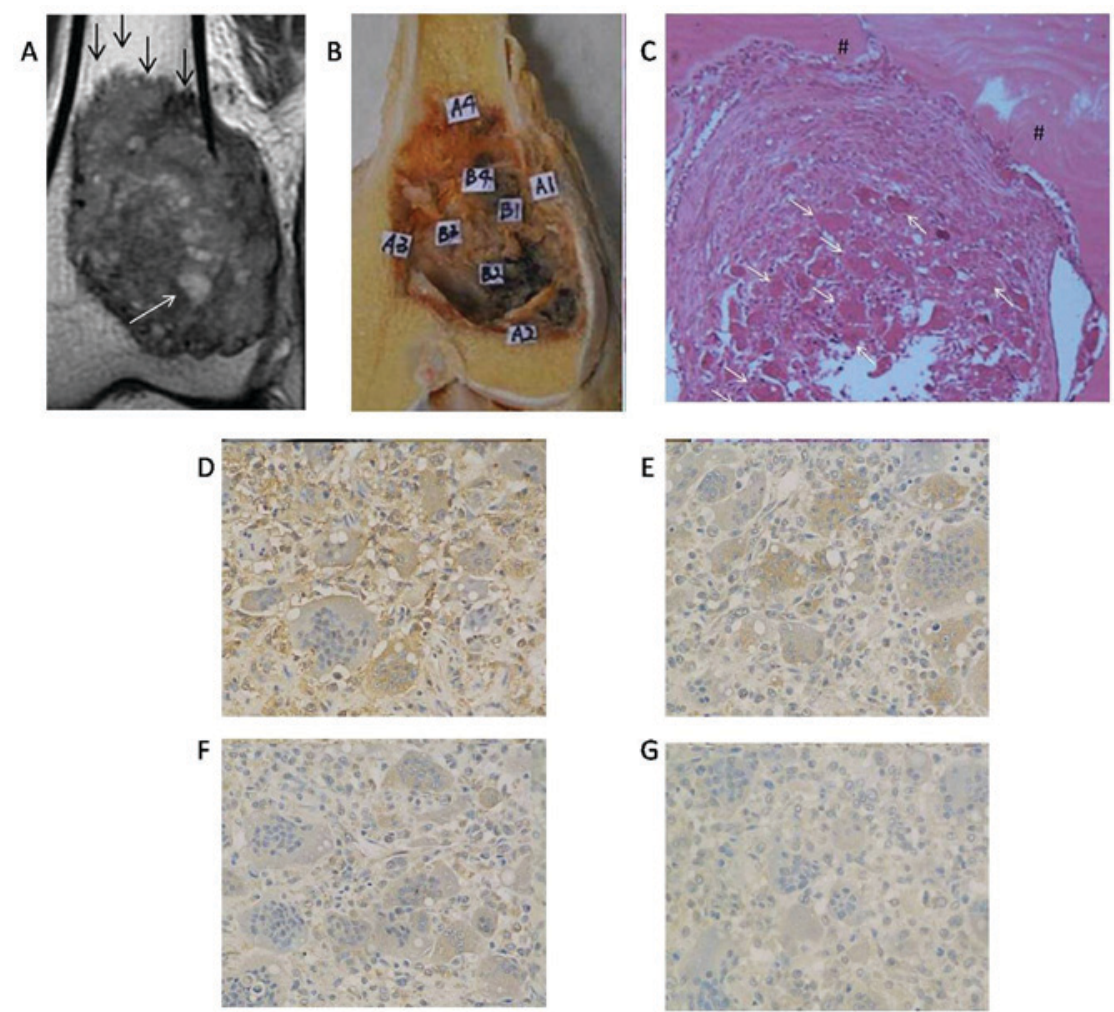

Figure 2. Representative images of a case of giant cell tumor of bone treated with en bloc resection. (A) Coronal T2-weighted imaging revealed a heterogeneous area of intermediate-high signal intensity, the 'paint brush borders' sign (indicated by black arrows) and cystic changes (high signal intensity indicated by white arrow), as well as involvement of adjacent soft tissue. (B) A sectioned and processed tissue sample for MMP-9 staining. (C) Hematoxylin-eosin staining photomicrograph equivalent to area A4 of Fig. 3B, demonstrating the tumor histology with typical multinuclear giant cells (indicated by white arrows) among numerous mononuclear cells protruding toward the bone tissue (indicated by ${ }^{("}$ '). Magnification, x100. (D) Diffuse strong receptor activator of nuclear factor- $\mathrm{kB}$ ligand expression (IHC grade 3). (E) Diffuse moderate MMP-9 expression (IHC grade 2). (F) Diffuse moderate vascular endothelial growth factor expression (IHC grade 2). (G) Diffuse weak receptor activator of nuclear factor-kB expression (IHC grade 1). x200, magnification. MMP-9, matrix metalloproteinase 9; IHC, immunohistochemistry.
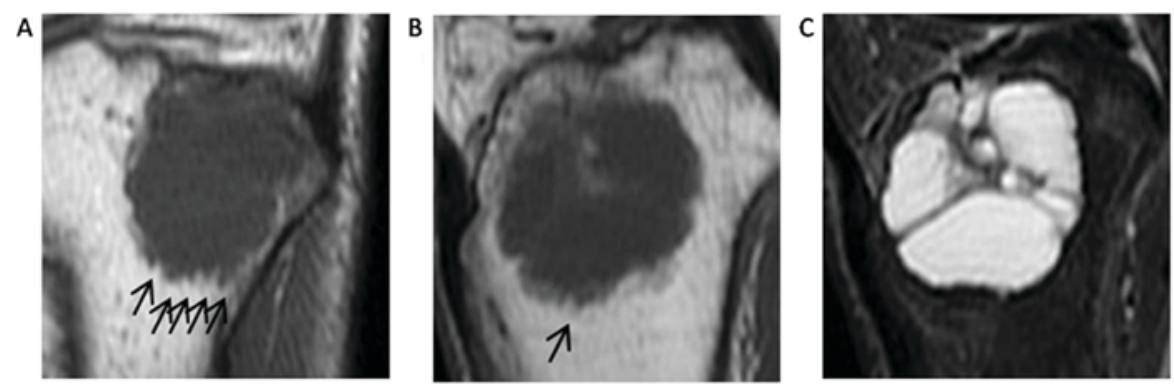

Figure 3. Representative magnetic resonance images of a case of GCTB in the proximal tibia treated with curettage. No sign of recurrence was evident 10 years after curettage. (A) Coronal and (B) sagittal T1-weighted images reveal the 'paint brush borders' sign (indicated by black arrows) protruding toward the bone from the edge of the GCTB. (C) A sagittal fat-suppressed T2-weighted image demonstrates minimal and limited peritumoral edema, classified as grade B in the present study. GCTB, giant cell tumor of bone.

tissue invasion was also revealed to be associated with local recurrence $\left(\chi^{2}=4.935 ; \mathrm{P}<0.05\right)$. Neither peritumoral edema, nor MMP-9, RANKL or VEGF expression were associated with local recurrence $(\mathrm{P}>0.05$; Table IV).

Analysis of confounding factors. A total of 30/33 patients who had undergone additional adjuvant treatment were successfully followed up. There was no significant difference in the recurrence rates of patients treated with or without additional adjuvant therapy $(\mathrm{P}>0.05)$. Furthermore, no significant differences were observed in the recurrence rates of patients enrolled between January 2005 and January 2010, and those enrolled between January 2010 and October 2015 $(\mathrm{P}>0.05$; Table IV).

Confirmation of preoperative MRI features with pathological analysis. Gross pathological sections from patients treated with en bloc resection were visually matched to the acquired MRI scans. The lengths of the protrusions of GCTB varied between 1.5 and $3.6 \mathrm{~mm}$ on the MRI scans. In the patients treated with en bloc resection, the lengths of the protrusions varied between 1.2 and $3.3 \mathrm{~mm}$ in gross pathological sections, while the MRI scans exhibited lengths between 1.6 and $3.2 \mathrm{~mm}$. A number of multinucleated giant cells uniformly distributed amongst 

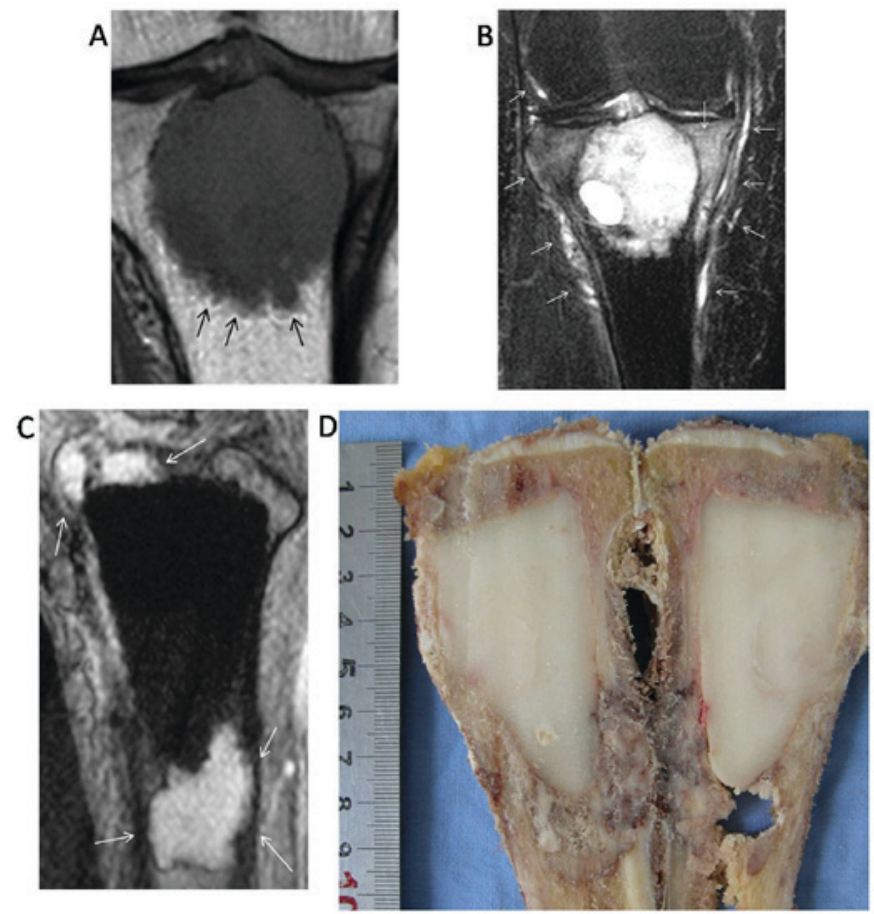

Figure 4. Representative images of a case of GCTB in the proximal tibia The patient experienced knee pain for 6 months and was treated with curettage. Recurrence was confirmed after 1 year of follow-up. (A) Coronal T1-weighted image demonstrating 'paint brush borders' sign-like irregular margins protruding toward the bone (indicated by black arrows). (B) Coronal fat-suppressed T2-weighted image demonstrating massive peritumoral edema and joint effusion (indicated by white arrows), which was classified as grade $\mathrm{A}$ in the present study. (C) Sagittal T2-weighted image demonstrating a homogeneous high signal intensity area (indicated by white arrows), suggesting local recurrence in the region of the penetrating irregular margins. (D) The surgical specimen was dissected to confirm the conclusions made from the sagittal images, and a recurrent tumor around bone cement was identified. GCTB, giant cell tumor of bone.

monocytes were observed in the protrusions (Figs. 2 and 5), and the 'paint brush borders' sign identified by preoperative MRI was also observable in the gross pathological sections (Fig. 5). Furthermore, the cystic changes and adjacent soft tissue invasion observed by MRI were visually matched to tissue samples corresponding to the GCTB coronal sections. Unfortunately, edema could not be evaluated pathologically as the sections were dehydrated.

\section{Discussion}

The primary purpose of the present study was to investigate the association between RANK, RANKL, VEGF and MMP-9 protein expression and preoperative MRI features identified in patients with GCTB. An association between MMP-9 and the 'paint brush borders' sign or cystic changes observable on MRI was demonstrated. Furthermore, specific preoperative MRI features were identified as prognostic factors in predicting local recurrence of GCTB.

The MMPs are a family of enzymatic proteins that are often overexpressed in the tumor microenvironment (24). MMP-9 is regarded as a gelatinase $\mathrm{B}$, type IV collagenase and is highly expressed during the early stages of osteoclast development, as well as in mature osteoclasts that resorb bone (25). The primary function of MMP-9 in GCTB is to stimulate bone re sorption by giant cells (1). In the present clinical study of preoperative MRI features, the 'paint brush borders' sign was visually matched with the corresponding gross pathological sections (Fig. 5). The pathological basis of the "paint brush borders' sign on preoperative MRI has been identified as an invasion of the bone around the lesions. Therefore, it has been hypothesized that the 'paint brush borders' sign may be indicative of osteolytic destruction. The present study revealed an association between the expression of MMP-9 and the 'paint brush borders' sign, which may contribute to the characterization of the molecular basis of this feature of the GCTB border.

To the best of our knowledge, the present study was the first to identify the 'paint brush borders' sign as a prognostic factor for local recurrence of GCTB following curettage. The success of intralesional surgical treatments is limited by the associated high rate of local recurrence. The present study revealed that GCTB tumors exhibiting the "paint brush borders' sign on preoperative MRI scans were more likely to recur following intralesional curettage.

The pathological basis of the 'paint brush borders' sign was investigated using tissue sections. Invasion of the bone around GCTB may be interpreted as an identifying feature of residual tumor $(9,10)$. Penetrating tumor margins may reduce the effect of intralesional surgical procedures. The present study provided evidence that supports the view that residual tumors may be responsible for local recurrence, and revealed the 'paint brush borders' sign as a risk factor for local recurrence of GCTB. This suggested that surgeons should increase their awareness of the 'paint brush borders' sign, and should pay more attention to ensure thorough elimination of residual tumors in regions of irregular protrusions by using more aggressive interventions. In the present study, the lengths of the protrusions observable by MRI were consistent with those measured in gross pathological sections. It should be emphasized that a more detailed assessment of the location of the penetrating irregular margins may aid in guiding aggressive surgical treatment. The present study suggested that thorough evaluation of preoperative MRI features may be useful in guiding surgical treatment and reduce local relapse.

MMPs are capable of degrading the entire extracellular matrix (26). In the present study, MMP-9 protein expression was demonstrated to be associated with the presence of cystic changes identified by preoperative MRI. Furthermore, cystic changes were identified as an independent risk factor for local recurrence. Based on these results, it is speculated that GCTB tumors exhibiting elevated MMP-9 protein expression levels maybe more prone to cystic changes. The recurrence rate in patients with GCTB with cystic changes was $59.46 \%$, whereas recurrence developed in only 1 case $(5.56 \%)$ in which cystic changes were not observed. This suggests differing properties in tumors with and without cystic changes. MRI is an important tool for identifying tumor properties, including cystic changes, which are associated with a poor prognosis.

A study on sacral chordoma demonstrated that positive expression of MMP-9 may be indicative of local recurrence (27). However, in the present study, high expression of MMP-9 was not positively associated with local recurrence, nor was expression of VEGF, RANK or RANKL. These negative results 
Table IV. Predicting local recurrence of GCTB and confounding variable analysis.

A, Preoperative MRI features $(n=55)$.

\section{Local recurrence}

Variable

Yes $(n=23)$

No $(n=32)$

$\chi^{2}$

P-value

Peritumoral edema

A

15

17

0.804

0.37

B

8

15

Cystic change

Yes

22

15

14.461

0.02

No

1

17

'Paint brush borders' sign

Yes

16

5

16.496

$<0.01$

No

7

27

Adjacent soft tissue invasion ${ }^{\mathrm{a}}$

Yes

20

19

4.935

0.04

No

3

13

Confounding variable analysis

Application of phenol

Applied

12

18

0.090

0.77

Not applied

$11 \quad 14$

Study span (January 2005 to October 2015)

First 5 years

11

13

0.282

0.56

Last 5 years

12

19

B, Immunohistochemical analysis $(\mathrm{n}=22)$.

\begin{tabular}{|c|c|c|c|c|}
\hline \multirow[b]{2}{*}{ Variable } & \multicolumn{2}{|c|}{ Local recurrence } & \multirow[b]{2}{*}{$\chi^{2}$} & \multirow[b]{2}{*}{ P-value } \\
\hline & Yes $(n=11)$ & No $(n=11)$ & & \\
\hline \multicolumn{5}{|l|}{ RANKL } \\
\hline High-grade & 7 & 4 & & 0.40 \\
\hline Low-grade & 4 & 7 & & \\
\hline \multicolumn{5}{|l|}{ RANK } \\
\hline High-grade & 4 & 1 & & 0.32 \\
\hline Low-grade & 7 & 10 & & \\
\hline \multicolumn{5}{|l|}{ VEGF } \\
\hline High-grade & 6 & 4 & & 0.67 \\
\hline Low-grade & 5 & 7 & & \\
\hline \multicolumn{5}{|l|}{ MMP-9 } \\
\hline High-grade & 6 & 2 & & 0.18 \\
\hline Low-grade & 5 & 9 & & \\
\hline
\end{tabular}

${ }^{\mathrm{a}} \mathrm{P}<0.05$. GCTB, giant cell tumor of bone; MRI, magnetic resonance imaging; IHC, immunohistochemistry; RANKL, receptor activator of nuclear

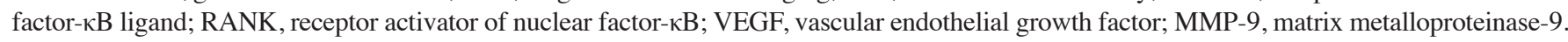

may be due to the limited sample size. A number of studies have demonstrated that co-expression of VEGF and MMP-9 is correlated with angiogenesis and invasiveness of human bone tumors $(13,27-29)$. In the present study, MMP-9 protein expression was associated with that of VEGF and RANKL (Table III). The activation of nuclear factor- $\kappa \mathrm{B}$ signaling may activate MMP expression in the tumor and surrounding stromal cells (29). Therefore, the upregulation of MMP-9 may 

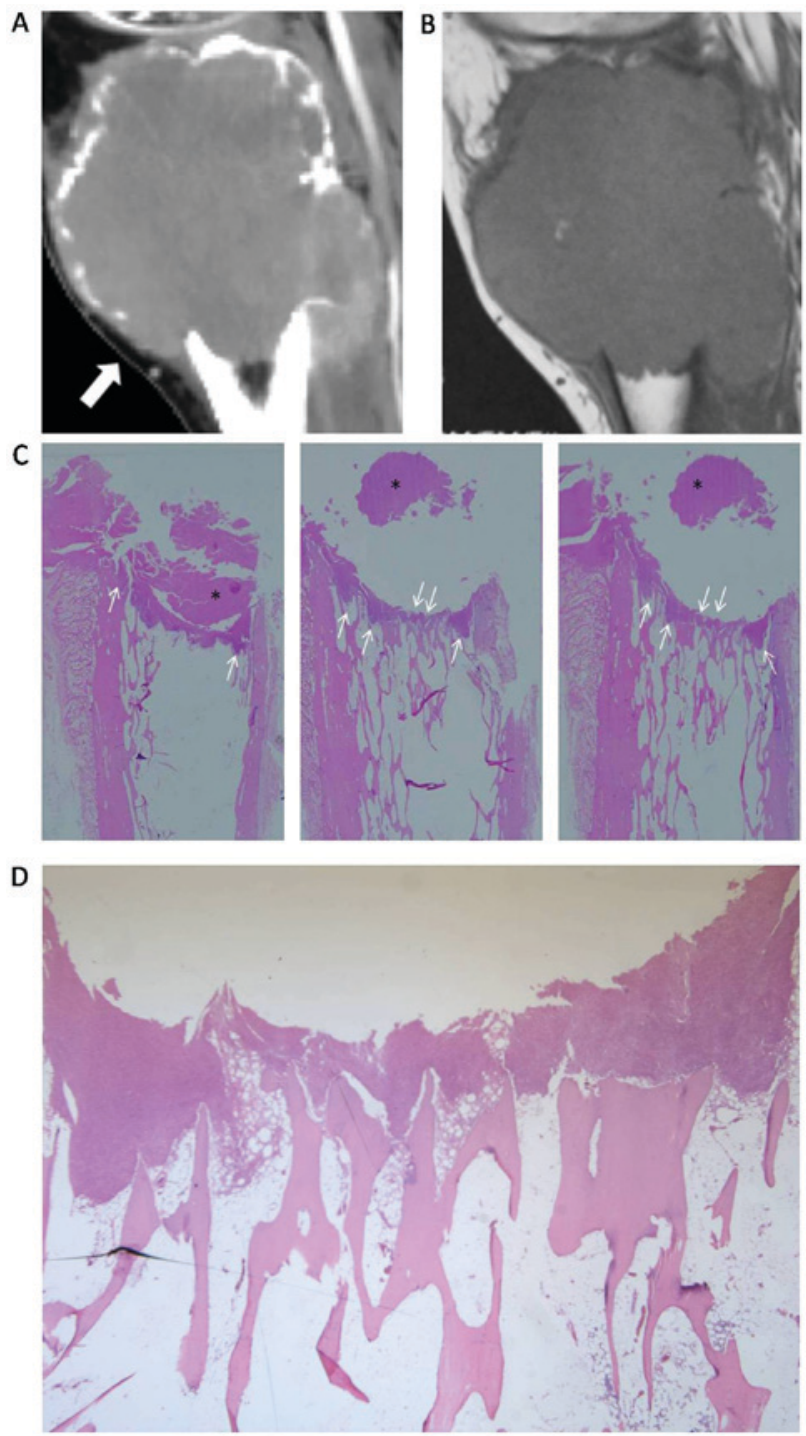

Figure 5. Representative images of a case of GCTB of a 24-year old female, in whom the treatment was delayed for 7 months due to pregnancy. Subsequently, en bloc resection was performed. Associations between the imaging conclusions and gross pathological conclusions were investigated. (A) Sagittal computerized tomography image demonstrating loss of bone cortex (thick white arrow) and soft tissue extension. (B) Sagittal T1-weighted image demonstrating protrusions (indicated by black arrows) on the lower margin of the GCTB tumor. (C) Images of sagittal gross pathological sections (original magnification, x100) exhibiting protrusions (indicated by white arrows) of the tumor margin (indicated by ${ }^{{ }^{*} \prime}$ ). (D) Photomicrograph of the gross pathological hematoxylin-eosin staining (original magnification, x25), indicating protrusions (white arrows) from the tumor margin, suggesting invasion of the bone (indicated by "\#').

be triggered by RANKL, which is expressed by spindle-like stromal cells in GCTB. Therefore, despite the lack of association with preoperative MRI features, the expression of RANKL in GCTB requires further investigation. Denosumab, a human monoclonal antibody that inhibits RANKL, has been demonstrated to be safe and effective in the treatment of GCTB (30).

Our previous study (17) demonstrated the role of soft tissue masses in the prognosis of GCTB. In the present study, a similar conclusion was made regarding adjacent soft tissue invasion. If the surgeons are able to identify the window of poor bone cortex integrity to perform the surgery in these patients with adjacent soft tissue invasions, it would theoretically decrease the contribution of adjacent soft tissue invasion to the risk of local recurrence.
Peritumoral edema was not identified as a predictor of local recurrence in the present study. Peritumoral edema is a reaction to tumor activity and is associated with its aggressiveness and poor prognosis (31). It is speculated that the unexpected results of the present study may be due to other confounding factors. For example, the use of phenol to prevent local relapse is controversial (9), and it may be considered a confounding factor. Furthermore, surgeons have always aimed to improve the surgical procedure in the long run, thereby lowering the recurrence rate. However, the rates of local recurrence in the first and last five years were 45.83 and $38.71 \%$, respectively, which are not significantly different.

The strengths of the present study include that it was standardized to patients with GCTB located around the knee. To exclude the impacts of pathological fracture (32) and soft tissue masses (17) on predicting local recurrence, the present study did not include patients with GCTB with evidence of these conditions.

As peritumoral edema was demonstrated in the majority of study patients, it was predicted that it was associated with recurrence. However, the classification of peritumoral edema may have been inaccurate or subjective. Furthermore, as the pathological sections were dehydrated, it was not possible to pathologically confirm edema. Additionally, the number of patients was relatively small to allow for IHC analysis. To the best of our knowledge, GCTB usually recurs within 2 years (33-35). However, as 3 patients in the present study developed recurrence after $\geq 2$ years of follow-up, the possibility of recurrence in other patients cannot be excluded without a longer period of follow-up.

In conclusion, IHC staining of MMP-9 was demonstrated to be associated with preoperative MRI features of the 'paint brush borders' sign and cystic changes, which, coupled with adjacent soft tissue invasion, were identified as risk factors for local recurrence of GCTB. These results may contribute to the prediction of local recurrence of GCTB, and may provide further insight into these preoperative features and expression patterns. The present study indicated that increased awareness and meticulous attention of surgeons, along with thorough preoperative MRI analysis, are important to reduce local recurrence of GCTB.

\section{Acknowledgements}

Not applicable.

\section{Funding}

The present study received funding from the Science and Technology Commission of Shanghai Municipality (grant no. 134119a2402).

\section{Availability of data and materials}

The analyzed data sets generated during the present study are available from the corresponding author, on reasonable request.

\section{Authors' contributions}

The integrity of entire study was guaranteed by XD. The study designed by XD and YH. The literature was researched by $\mathrm{YH}$ and YZ. The clinical studies were performed by $\mathrm{YH}$ and YZ. The experimental studies were performed by FY, JW, QZ 
and JL. Data was acquired by JW, YH and YZ. Imaging interpretation was performed by XD, LD, YH and YZ. Statistical analyses were performed by $\mathrm{YH}$ and $\mathrm{YZ}$. The manuscript was prepared by $\mathrm{YH}$. The manuscript was edited by $\mathrm{YH}$ and YZ. The manuscript was reviewed by XD and JZ. JX made substantial contributions to conception and design.

\section{Ethics approval and consent to participate}

The present study was approved by the institutional review board of Ruijin Hospital, Shanghai Jiao Tong University School of Medicine (Shanghai, China). Written informed consent was obtained from all patients prior to enrolment in the study.

\section{Consent for publication}

The participants of the present study provided written informed consent for publication.

\section{Competing interests}

The authors declare that they have no competing interests.

\section{References}

1. Cowan RW and Singh G: Giant cell tumor of bone: A basic science perspective. Bone 52: 238-246, 2013

2. Nishimura $M$, Yuasa $K$, Mori $K$, Miyamoto $N$, Ito $M$, Tsurudome M, Nishio M, Kawano M, Komada H, Uchida A and Ito Y: Cytological properties of stromal cells derived from giant cell tumor of bone (GCTSC) which can induce osteoclast formation of human blood monocytes without cell to cell contact. J Orthop Res 23: 979-987, 2005.

3. Siddiqui MA, Seng C and Tan MH: Risk factors for recurrence of giant cell tumours of bone. J OrthopSurg (Hong Kong) 22: 108-110, 2014.

4. van der Heijden L, Dijkstra PD, van de Sande MA, Kroep JR, Nout RA, van Rijswijk CS, Bovée JV, Hogendoorn PC and Gelderblom H: The clinical approach toward giant cell tumor of bone. Oncologist 19: 550-561, 2014.

5. Hu P, Zhao L, Zhang H, Yu X, Wang Z, Ye Z, Wu S, Guo S, Zhang G, Wang J, et al: Recurrence rates and risk factors for primary giant cell tumors around the knee: A multicentre retrospective study in china. Sci Rep 6: 36332, 2016.

6. Zambo I and Vesely K: WHO classification of tumours of soft tissue and bone 2013: The main changes compared to the 3rd edition. Cesk Patol 50: 64-70, 2014 (In Czech).

7. Enneking WF: A system of staging musculoskeletal neoplasms. Clin Orthop Relat Res: 9-24, 1986.

8. Wülling M, Engels C, Jesse N, Werner M, Delling G and Kaiser E: The nature of giant cell tumor of bone. J Cancer Res Clin Oncol 127: 467-474, 2001.

9. Klenke FM, Wenger DE, Inwards CY, Rose PS and Sim FH: Giant cell tumor of bone: Risk factors for recurrence. Clin Orthop Relat Res 469: 591-599, 2011.

10. Arbeitsgemeinschaft Knochentumoren, Becker WT, Dohle J, Bernd L, Braun A, Cserhati M, Enderle A, Hovy L, Matejovsky Z, Szendroi M, et al: Local recurrence of giant cell tumor of bone after intralesional treatment with and without adjuvant therapy. J Bone Joint Surg Am 90: 1060-1067, 2008.

11. Masui F, Ushigome $S$ and Fujii K: Giant cell tumor of bone: An immunohistochemical comparative study. Pathol Int 48: 355-361, 1998.

12. Wu Z, Yin H, Liu T, Yan W, Li Z, Chen J, Chen H, Wang T, Jiang Z, Zhou W and Xiao J: MiR-126-5p regulates osteoclast differentiation and bone resorption in giant cell tumor through inhibition of MMP-13. Biochem Biophys Res Commun 443: 944-949, 2014.

13. Kumta SM, Huang L, Cheng YY, Chow LT, Lee KM and Zheng MH: Expression of VEGF and MMP-9 in giant cell tumor of bone and other osteolytic lesions. Life Sci 73: 1427-1436, 2003.

14. Quattrini I, Pollino S, Pazzaglia L, Conti A, Novello C, Ferrari C, Pignotti E, Picci P and Benassi MS: Prognostic role of nuclear factor/IB and bone remodeling proteins in metastatic giant cell tumor of bone: A retrospective study. J Orthop Res 33: 1205-1211, 2015.
15. Tang T,Zhang G,Lau CP, Zheng LZ, Xie XH, Wang XL, Wang XH, He K, Patrick Y, Qin L and Kumta SM: Effect of water-soluble P-chitosan and S-chitosan on human primary osteoblasts and giant cell tumor of bone stromal cells. Biomed Mater 6: 015004, 2011.

16. Wang CS, Lou JH, Liao JS, Liao JS, Ding XY, Du LJ, Lu Y, Yan L and Chen KM: Recurrence in giant cell tumour of bone: Imaging features and risk factors. Radiol Med 118: 456-464, 2013.

17. Chen L, Ding XY, Wang CS, Si MJ, Du LJ, Zhang WB and Lu Y: In-depth analysis of local recurrence of giant cell tumour of bone with soft tissue extension after intralesional curettage. Radiol Med 119: 861-870, 2014

18. Klenke FM, Wenger DE, Inwards CY, Rose PS and Sim FH: Recurrent giant cell tumor of long bones: Analysis of surgical management. Clin Orthop Relat Res 469: 1181-1187, 2011.

19. Prosser GH, Baloch KG, Tillman RM, Carter SR and Grimer RJ: Does curettage without adjuvant therapy provide low recurrence rates in giant-cell tumors of bone? Clin Orthop Relat Res: 211-218, 2005.

20. Wang $\mathrm{H}$, Wan $\mathrm{N}$ and $\mathrm{Hu} \mathrm{Y}$ : Giant cell tumour of bone: A new evaluating system is necessary. Int Orthop 36: 2521-2527, 2012.

21. de Moraes M, de Matos FR, de Souza LB, de Almeida Freitas R and de Lisboa Lopes Costa A: Immunoexpression of RANK, RANKL, OPG, VEGF and vWF in radicular and dentigerous cysts. J Oral Pathol Med 42: 468-473, 2013.

22. Kanematsu M, Semelka RC, Leonardou P, Mastropasqua M, Armao D, Vaidean G, Firat Z and Woosley JT: Angiogenesis in hepatocellular nodules: Correlation of MR imaging and vascular endothelial growth factor. J Magn Reson Imaging 20: 426-434, 2004.

23. He Y, Zhang J and Ding X: Prognosis of local recurrence in giant cell tumour of bone: What can we do? Radiol Med 122: 505-519, 2017.

24. Chambers AF and Matrisian LM: Changing views of the role of matrix metalloproteinases in metastasis. J Natl Cancer Inst 89: 1260-1270, 1997.

25. Reponen P, Sahlberg C, Munaut C, Thesleff I and Tryggvason K: High expression of $92-\mathrm{kD}$ type IV collagenase (gelatinase B) in the osteoclast lineage during mouse development. J Cell Biol 124: 1091-1102, 1994.

26. Lynch CC and Matrisian LM: Matrix metalloproteinases in tumor-host cell communication. Differentiation 70: 561-573, 2002.

27. Chen KW, Yang HL, Lu J, Wang GL, Ji YM, Wu GZ, Zhu LF, Liu JY, Chen XQ and Gu YP: Expression of vascular endothelial growth factor and matrix metalloproteinase-9 in sacral chordoma. J Neurooncol 101: 357-363, 2011.

28. Kaya M, Wada T, Kawaguchi S, Nagoya S, Yamashita T, Abe Y, Hiraga H, Isu K, Shindoh M, Higashino F, et al: Increased pre-therapeutic serum vascular endothelial growth factor in patients with early clinical relapse of osteosarcoma. Br J Cancer 86: 864-869, 2002.

29. Shuman Moss LA, Jensen-Taubman S and Stetler-Stevenson WG: Matrix metalloproteinases: Changing roles in tumor progression and metastasis. Am J Pathol 181: 1895-1899, 2012.

30. Chawla S, Henshaw R, Seeger L, Choy E, Blay JY, Ferrari S, Kroep J, Grimer R, Reichardt P, Rutkowski P, et al: Safety and efficacy of denosumab for adults and skeletally mature adolescents with giant cell tumour of bone: Interim analysis of an open-label, parallel-group, phase 2 study. Lancet Oncol 14: 901-908, 2013.

31. Wu CX, Lin GS, Lin ZX, Zhang JD, Chen L, Liu SY, Tang WL, Qiu XX and Zhou CF: Peritumoral edema on magnetic resonance imaging predicts a poor clinical outcome in malignant glioma. Oncol Lett 10: 2769-2776, 2015.

32. O'Donnell RJ, Springfield DS, Motwani HK, Ready JE, Gebhardt MC and Mankin HJ: Recurrence of giant-cell tumors of the long bones after curettage and packing with cement. J Bone Joint Surg Am 76: 1827-1833, 1994.

33. Scully SP, Mott MP, Temple HT, O'Keefe RJ, O'Donnell RJ and Mankin HJ: Late recurrence of giant-cell tumor of bone. A report of four cases. J Bone Joint Surg Am 76: 1231-1233, 1994.

34. Lausten GS, Jensen PK, Schiodt T and Lund B: Local recurrences in giant cell tumour of bone. Long-term follow up of 31 cases. Int Orthop 20: 172-176, 1996

35. Kivioja AH, Blomqvist C, Hietaniemi K, Trovik C, Walloe A, Bauer HC, Jorgensen PH, Bergh P and Follerås G: Cement is recommended in intralesional surgery of giant cell tumors: A Scandinavian Sarcoma Group study of 294 patients followed for a median time of 5 years. Acta Orthop 79: 86-93, 2008. 